\section{俩 Heighten Science \\ P U B L I C I T I O N S Corporation \\ ISSN \\ 2575-0143}

\title{
Not-motorized Implantable Cardiac Assistance (NICA): Hemodynamic concepts and clinical perspectives
}

\author{
Philippe Caimmi ${ }^{1 *}$, Martinelli GL ${ }^{2}$ and Barbieri ${ }^{1}$ \\ ${ }^{1}$ R\&I and Clinical Trial Center, University Hospital of Novara, Italy \\ ${ }^{2}$ Cardiac Surgery, Clinica San Gaudenzio, Novara, Italy
}

\begin{abstract}
*Address for Correspondence: Philippe Caimmi, R\&I and Clinical Trial Center, University Hospital of Novara, Italy, Tel: 0039 3472329376; Email: philippe.caimmi@maggioreosp.novara.it; pcaimmi@gmail.com
\end{abstract}

Submitted: 03 June 2019

Approved: 12 June 2019

Published: 13 June 2019

Copyright: @ 2019 Caimmi P, et al. This is an open access article distributed under the Creative Commons Attribution License, which permits unrestricted use, distribution, and reproduction in any medium, provided the original work is properly cited

Check for updates

\section{Abstract}

The considerable improvements in cardiac support systems technologies have not solved until now the problem of connecting the cardiac assistance devices (CAD) to external energy sources, which makes these Patients at risk of lethal infections and dependent on external batteries with few hours of autonomy. Authors illustrate and discuss the hemodynamic concepts and clinical that underlie the mechanics of the first not-motorized implantable cardiac assistance device (NICA).

\section{Introduction}

The increase in the average age is an important cause of the continuous increase in heart failure in west countries. Cardiac transplantation remains the most effective therapy in the forms of ventricular failure that are refractory to medical therapy but the insufficient availability of donors prevents the overall solution of this important public health problem. For these reasons, in last decades mechanical assistance has become a central tool in the treatment of severe heart failure, including bridge to recovery, bridge to transplant and destination therapy [1]. However, the considerable improvements in cardiac support systems technologies have not solved the problem of connecting the CAD to external energy sources until now, which makes these Patients at risk of lethal infections and dependent on external batteries with few hours of autonomy. In addition all the available systems have limitations concerning the implantation technique, the interaction with the blood, the safety of the patient in case of mechanical failure [2-4]. In this article, the authors will illustrate and discuss the concepts that underlie the mechanics of a new and original not-motorized implantable circulatory assistance device (NICA) [5], would seem to be a possible solution to the problems outlined above.

\section{Hemodynamic concepts of NICA}

The not-motorized implantable circulatory assistance device (NICA) is described in detail by authors in a previous paper and patent [5,6]. It is an aortic-aortic bypass of the descendent thoracic aorta including an elastically expandable chamber (EEC) as described in figure 1.

NICA by mean of its EEC that is connected in parallel to the thoracic descendent aorta acts as a significant augmentation of compliance and consequently reduction of resistances in the proximal thoracic aorta according to the Poiseuille's law. If we ideally take into account the passive hemodynamic behavior of assistant devices, ie when not active and not valved, it is clear that the aorto-aortic bypass solution (AAB) is in itself 


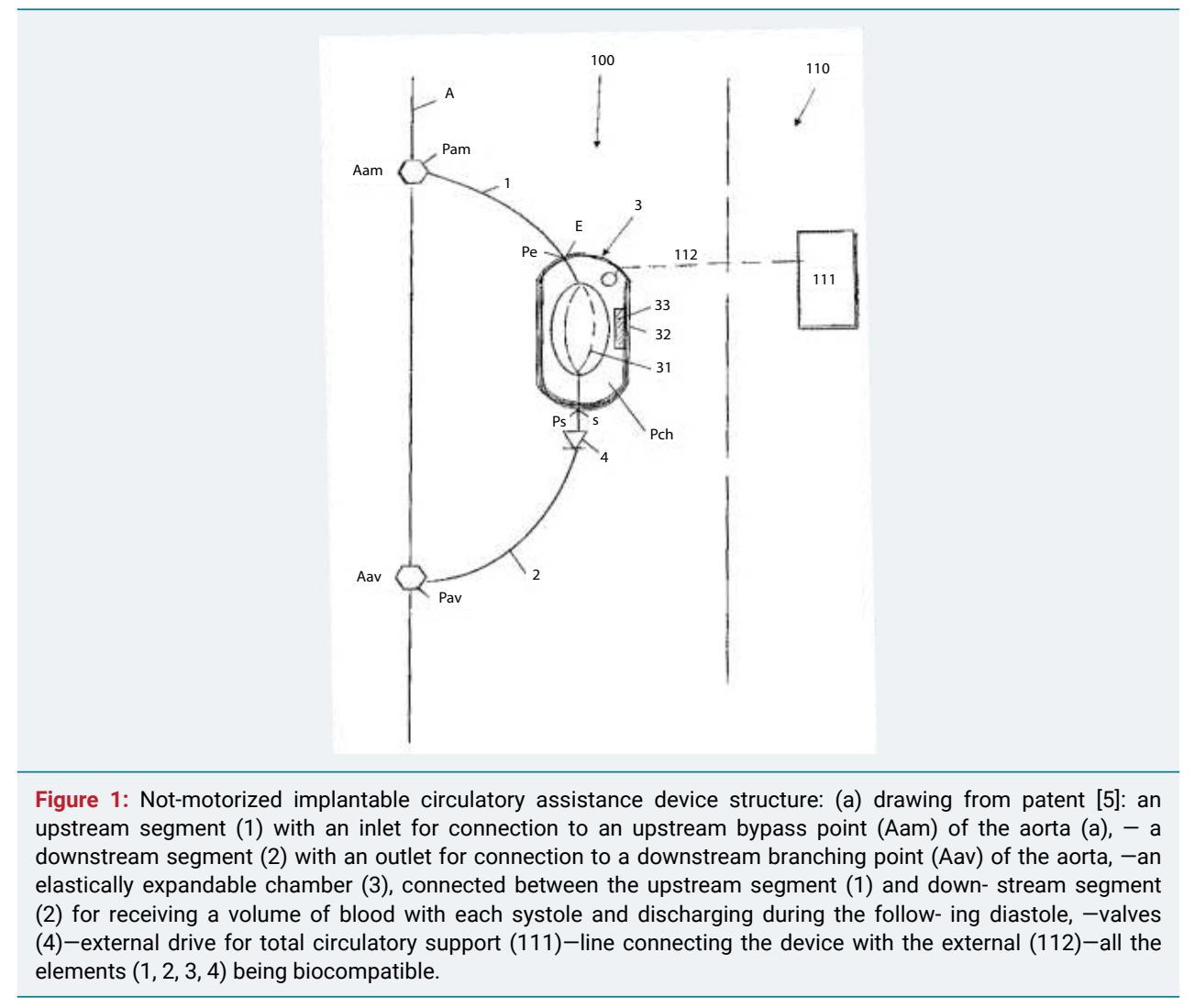

an advantage over ventricular-aortic bypass-related assistant devices (VAB). This is because $\mathrm{VAB}$ without valve reduces cardiac output by behaving like a massive aortic insufficiency. AAB also without a valve reduces aortic resistance without creating the VTR ventricular overload and therefore increases cardiac output per se. In reality this consideration is applicable only for pulsatile assistant devices (PD) but not in those not pulsatile assistant devices (NPD= axial or centrifugal pumps) where the continuity equation is not applicable due to the interruption of the flow which for the structure of NPD. Therefore NPDs do not have a passive hemodynamic component. This makes them much more dangerous in case of mechanical failure. Another consideration that can be made is that the PDs, having their own chamber, can work in counter-impulse. Consequence of this is the fact that the assistance in counter pulsation has no impact on the mobility of the aortic valve which can open and close without obstacles. In NPDs, on the other hand, for greater the assistance rate it can be observed a longer aortic valve closing time, and this greatly increases the embolic risk. Within the PD, the use of the $\mathrm{AAB}$ allows to implement the counter-pulsed assistance in the absence of valve ducts, which is not possible for PDs using VAB. Thus the combination of PD and $\mathrm{AAB}$ represents the option for cardiac assistance with the lowest inertia and greater passive effect and therefore requiring the lowest energy consumption to exercise active support. The inclusion in the AAB of an EEC specifically designed with high efficiency elastomer, with design and volume optimized to maximize the compliance effect of $\mathrm{AAB}$ and the elastic return of EEC. As reported by the authors in previous publication [1] the actual support of NICA varies inversely to the peripheral resistance (from 2100 dyn s / cm5 to 700 dyn s / cm5 respectively from $+71 \%$ to $+25 \%$ ) and this according to its passive hemodynamic model [6]. Naturally NICA can only perform partial assistance. In consideration of these preliminary data it is hypothesizable that NICA can be used in patients with an ejection fraction $(E F) \geq 30 \%$. Because surgical implant of a partial support device is not acceptable without the opportunity to shift to a total support in case of acute severe heart failure, authors also developed an external pneumatic pump connected to NICA that can be used when a total circulatory support is required, as in the case of a severe acute heart failure (EF $\leq 30 \%)$. It represents "de 
facto" as a bridge to the partial recovery that can be weaned when the recovered EF becomes $\geq 30 \%$. The passive hemodynamic performances of NICA indicate that the possible association of an active function by means of an external drive would require much less energy than other assistance systems. Lower energy consumption is of great importance in the miniaturization of external drives and batteries.

\section{Clinical speculations}

The device alone is not subject to infective contamination of the connection cable to the external power source. The implantation of the device is done without cardiopulmonary bypass (CPB). The embolic risk of the device should be reduced by the shape of the EEC that has the inflow and the outflow directly in line with the main axis. In addition, since the conduit of outflow is anastomosed in the descendent thoracic aorta, the risk of stroke is minimized to the only possibility of peripheral embolism. Because of its extra-cardiac position, the device can be combined with other procedures for heart failure as cardiac resynchronization therapy devices, Mitraclip and transcatheter aortic valve implantation. In the case of adequate recovery of cardiac function, the device can stay in place with a mild thromboembolic prevention therapy or easily removable without the use of CPB. In particular, unlike devices with apical ventricular connection, the removal is devoid of embolic risk and does not involve losing the function of the apical myocardium [6].

\section{Conclusion}

These considerations led our research group to conceive the possibility that a PD through an $\mathrm{AAB}$ could be implemented without an external drive. As mentioned in the introduction, the absence of an external drive eliminates one of the major limitations of cardiac assistant devices. The concepts of development and the hemodynamic behavior of NICA represent an interesting and original contribution to the development of more efficient assistance cardiac devices and opens a new point of view in favor of new pulsatile devices specifically developed to be placed in parallel to the descendent thoracic aorta.

\section{References}

1. Holman WL, Naftel DC, Eckert CE, Kormos RL, Goldstein DJ, et al. Durability of Left Ventricular Assist Devices: Inter-agency Registry for Mechanically Assisted Circulatory Support (INTERMACS) 2006 to 2011. J Thorac Cardiovasc Surg. 2013; 146: 437-441. Ref.: http://bit.ly/2WAZzlY

2. Krabatsch T, Schweiger M, Stepanenko A, Drews T, Potapov E, et al. Improvements in Implantable Mechanical Circulatory Support Systems: Literature Overview and Update. Herz. 2011; 36: 622-629. Ref.: http://bit.ly/2wNUOWP

3. Ujeyl A, Krüger M. Rise of the Machines? Left Ventricular Assist Devices for Treatment of Severe Heart Failure. Herz. 2015; 40: 972-979. Ref.: http://bit.ly/2lb95TZ

4. Englberger L, Reineke DC, Martinelli MV, Mohacsi P, Carrel TP, et al. Ventricular Assist Device: Possibilities of Long-Term Mechanical Circulatory Support. Ther Umsch. 72: 505-511. Ref.: http://bit.ly/2WtLX7a

5. EPO Patent Bibliographic Data. Dispositif D'assistance Circulatoire Implantable Non Motorise. Barbieri Galdino, France. 2017. Ref.: http://bit.ly/219T1BZ

6. Martinelli GL, Barbieri G, Caimmi P. A Not-Motorized Implantable Device for Partial Circulatory Support: A Proof of Concept Experiment. J Med Devices. 2018; 1-5. Ref.: http://bit.ly/2MJ6uF5 